\section{Bird communities}

Competition and the Structure of Bird Communities. By M. L. Cody. Pp. viii + 318. (Monographs in Population Biology, No. 7.) (Princeton University: New Jersey, July 1974.) \$6.60 cloth; $\$ 3.65$ paper.

CoDy's book is of more general interest than the title would indicate for he has attempted, with some success, to effect a synthesis between the data of field biologists and the niche and competition models of theoretical ecologists. It will be of interest even to ecologists who hate birds. Cody emphasises the importance of "natural experiments" in understanding community structure, and he makes informed comparisons of mainland and island bird communities, and of California chaparral and Chile matorral bird communities.

$\mathrm{He}$ presents a great deal of data that are consistent with the predictions of the theory of community structure developed by the late Robert MacArthur. For example, Cody finds that variation in foliage height diversity accounts for $81 \%$ of the variation in bird species diversity in eight North American bird communities (page 128). Cody's extensive field data point out some of the weaknesses of current theory: suppose that one can measure accurately the amount of competition for food, and that one can measure accurately the amount of competition for habitat. How can they be combined into one number that reflects the total amount of competition between two species? If competition along different niche axes (food, habitat, and so on) is independent, then the total amount of competition is the product of the competition coefficients along the different axes. This assumption of independence has usually been made by theoretical ecologists, but Cody found that addition, rather than multiplication of the competition coefficients along the different axes made for better predictions of community structure.

At times I found the book frustrating because Cody did not develop some of his insights more thoroughly. For example, Cody repeats and partially justifies one sacred cow of ecology'jack-of-all trades and master of none' (page 55), but on page 68 he shows that species that occupy a broad habitat range often eat a broad range of foods, and argues that this is optimal. Such a species would seem to be a 'jack-of-all trades and master of some'. Another example is his demonstration of character convergence, which he argues is a consequence of interspecific competition. Yet he does not discuss or test the model of Mac-
Arthur and Levins that predicts character convergence when a species invades a community in which resource overlap between species is high. Instead, Cody discusses the relationship with interspecific territoriality, but this seems to confuse cause and effect.

Readers not convinced that competition is a major factor in community organisation may not be persuaded by Cody's book. In some cases, alternative explanations are available for the phenomena Cody attributes to competition: for example, geographical separation of closely related species. Nevertheless, Cody presents a large diverse body of data consistent with competition theory. Any refutation of the importance of competition in determining community structure must account for the extensive observations Cody has made. David C. Culver

\section{Carbon and light}

Organic Photochemistry. By J. M. Coxon and B. Halton. (Cambridge Chemistry Texts). P. vii + 196. (Cambridge University: London, 1974.) $£ 4.20 ; \$ 13.00$.

Although several new books on organic photochemistry, aimed mainly at senior undergraduate and graduate students, have been published in the last two or three years the subject is undergoing such rapid expansion that new titles are always welcome. The book under review is divided into five chapters dealing with an introduction to photochemistry; intramolecular reactions of olefinic bonds; intramolecular reactions of carbonyl compounds; cycloaddition reactions; and substitution, oxidation, and reduction. The text is commendably free from typographical errors.

One of the many problems confronting authors in the area of organic photochemistry is the quantity of scientific literature to be covered. There is no possibility of encyclopaedic coverage in a small volume but the information cited should at least be correct. Unfortunately, this is not always so in this book. On page 140 it is stated that 2-methoxynaphthalene affords a $(2+2)$ dimer involving the 9,10 bonds, though, in fact, the dimer formed is of the $(4+4)$ type involving addition across the 1,4 sites of the substituted ring. This structure has been verified by X-ray analysis (Chem. Commun., 978; 1969).

On the whole the authors have kept this book up to date although, inevitably, since the publication date new results have out-dated some of the reaction mechanisms. The book is very readable and gives coverage suitable for the students at whom it is aimed.

\section{Ising model}

The Two-Dimensional Ising Model. By Barry M. McCoy and Tai Tsun Wu. Pp. xvi+418. (Harvard University: Cambridge, Massachusetts; Oxford University: London, February 1974.) $£ 12.50$.

THE two-dimensional Ising model gives a simplified description of a wide range of physical systems including fluids, magnets and binary alloys; without question it is the most thoroughly investigated model in statistical mechanics. Its main interest is that, though simply formulated, it has a surprising richness and complexity, and throws much light on the behaviour of systems undergoing a phase transition. In $1944 \mathrm{~L}$. Onsager performed the major feat of calculating the partition function of the square Ising lattice in zero field, and found some of its thermodynamic properties. The results profoundly changed the theory of phase transitions and critical behaviour.

Onsager's calculation and later investigations by others were notorious for their difficulty, and this made the study of the Ising model practically a branch of theoretical physics by itself. In 1962 the Dutch physicist P. W. Kasteleyn achieved considerable simplification by showing that the two-dimensional model in zero field is equivalent to the combinatorial problem of placing dimers on the bonds of the lattice such that each lattice point is covered by the end of one dimer, and counting the number of ways in which this is possible. This problem has a simple solution in terms of the Pfaffian of a matrix which can be evaluated without much difficulty. Over the last 12 years the method has become a powerful tool by which many more detailed properties of the model have been evaluated. The authors of the present monograph have made notable contributions in this field. Even though the method is simple in principle, research papers still tend to be hard reading because of their conciseness and the amount of detail. The aim of this book is to give an elaborate exposition of the dimer method and its application in various situations. It does not present a comprehensive survey of all the work which has been done on the two-dimensional Ising model. The authors have succeeded admirably in their limited aim and have given a careful exposition of the mathematical techniques involved and the results obtained. For those who want to be acquainted with the model and its history some existing reviews would seem preferable. But this book is recommended to anyone aspiring to research in the area and wishing to acquire a working knowledge of the dimer method. $\quad$ B. U. Felderhof 